\title{
REGIONAL VARIATIONS IN EXCITABILITY OF BARNACLE NEURONS ${ }^{1}$
}

\author{
VICTOR KRAUTHAMER ${ }^{2}$ AND WILLIAM N. ROSS ${ }^{3}$ \\ Department of Physiology, New York Medical College, Valhalla, New York 10595 and The Marine Biological Laboratory, \\ Woods Hole, Massachusetts 02543
}

Received June 16, 1983; Revised September 23, 1983; Accepted October 4, 1983

\begin{abstract}
Optical recording techniques using voltage-sensitive dyes were used to examine the initiation and propagation of action potentials within neurons of the supraesophageal ganglion of the giant barnacle, Balanus nubilus.

When a neuron was stimulated with current injection into the soma, the site of spike initiation, determined as the location with the earliest time-to-peak, was usually in the axon, 100 to $200 \mu \mathrm{m}$ from the soma. The soma spike was broader and often later, suggesting that the cell body was less excitable than the axon.

The action potential was narrowest at the site of initiation and then widened before propagating uniformly down the axon.

In most cases, somatically stimulated action potentials and electrotonic pulses propagated into the dendrites with little delay or change of shape, indicating that the electrotonic length of these processes was great.

Several different kinds of experiments indicated that some dendrites of these cells are excitable. These included the observations that $(a)$ spikes could be made to initiate earlier in the dendrites than in the axonal region to which they were connected, and $(b)$ action potentials sometimes decremented less than subthreshold pulses along dendritic processes.

However, in other cases a decline in amplitude and a widening of the action potential demonstrated passive propagation into the dendrites, suggesting that not all dendrites are equally excitable.
\end{abstract}

The regional electrical properties of neurons are key elements in determining how these cells integrate and transmit information. For example, the degree of excitability of dendrites determines whether the cell will integrate synaptic potentials over a small area or over the whole dendritic field. Similarly, the location of the spike initiation zone significantly affects the relative weight of different synaptic inputs. The values of the passive cable parameters of the membrane in different regions also may differ. In most cells, these regional properties have not been adequately determined, largely

\footnotetext{
${ }^{1}$ This work supported in part by United States Public Health Service Research Grant NS-016295 (W. N. R.), National Research Scientist Award Fellowship NS-06929 (V. K.), and an Irma T. Hirschl Career Scientist Award (W. N. R.). We wauld like to thank Nechama LasserRoss for most of the computer programming and L. B. Cohen for comments on the manuscript.

${ }^{2}$ Present address: Department of Physiology, Southeastern College of Osteopathic Medicine, North Miami Beach, FL 33162.

${ }^{3}$ To whom correspondence should be addressed, at Department of Physiology, New York Medical College, Valhalla, NY 10595.
}

because they have had to be deduced from indirect measurements.

Dendritic excitability, for example, has been inferred from intracellular recording from dendrites (Llinas and Nicholson, 1971; Wong et al., 1979; Llinas and Sugimori, 1980), by examination of somatic potential shapes (Spencer and Kandel, 1961; Takeda and Kennedy, 1965; Sandeman, 1969), and by extracellular recordings (Fatt, 1957; Llinas, 1975). Although it is unlikely that the conclusion that some dendrites are excitable is wrong, each of these methods has some limitations. First, intradendritic recordings from one position cannot determine the site of origin of the action potential since it is possible that a recorded spike propagated passively to the electrode position from the soma or axon. In experiments in which dendritic excitability has been inferred from somatic recordings there is the problem that the shapes of the recorded potentials are subject to other interpretations (see discussion in Llinas, 1975). The same limitation is found in analyzing extracellular potentials (Fatt, 1957; Nelson and Frank, 1964; Purpura, 1967). Whereas most experiments describing dendritic excitability have 
been done on vertebrate neurons (reviewed by Llinas, 1975), several groups (Takeda and Kennedy, 1965; Sandeman, 1969; Calabrese and Kennedy, 1974) have noted that some invertebrate processes might be excitable. Most of their conclusions were reached by the analysis of somatically recorded potential shapes. In addition, in these experiments, there was no morphological description of the cells to indicate the location of the excitable processes.

The site of spike initiation in a motoneuron axon has also been controversial. Pearson and Fourtner (1975) suggested on the basis of amplitude measurements that in cells of the cockroach metathoracic segment, this point was close to where the axon leaves the ganglion. Sandeman (1969) came to the same conclusion in examining crab motoneurons using extracellular recordings. He found that the initial axon segment, to which the dendritic processes connected, was inexcitable. However, Gwilliam and Burrows (1980) placed the initiation zone closer to the soma in locust metathoracic motoneurons. They used both multiple extracellular recordings and timing differences between two intracellular points in making their determination. Tauc (1962a, b), using three intracellular electrodes in Aplysia motoneurons, located this point outside the soma. But his spatial resolution was not fine enough to determine whether it was within the ganglion. In vertebrate motoneurons the initiation zone has been assigned to the axon hillock largely on the basis of potential shapes in the soma (Fuortes et al., 1957; however, see Furshpan and Furukawa, 1962).

We have examined the questions of dendritic excitability and spike initiation zone location in barnacle neurons using the optical techniques described in the previous paper (Ross and Krauthamer, 1984). 'This technique allows us to make simultaneous recordings of potentials from many identified positions on the same cell. By directly looking for the site of earliest time-to-peak and the shape of action potentials and comparing them with electrotonically spreading potentials, we can determine the initiation point for the spike and the excitability of different regions. Some of these results have been reported previously in abstract form (Krauthamer and Ross, 1982).

\section{Materials and Methods}

Most experiments were performed on three or four large neurons whose cell bodies lie on the anteromedial margin of the supraesophageal ganglion of Balanus nubilus. These cells have been called "marginal cells." They send large axons out of the ipsilateral antennular nerve and are thought to be motoneurons (G. F. Gwilliam, personal communication).

Our procedures were essentially as described in the previous paper (Ross and Krauthamer, 1984). The location of the optical recordings was determined in most cases by directly correlating the photodiode array position with the unfixed Lucifer Yellow-filled cell. In some cases, the fine processes were not visible in the unfixed tissue (Ross and Krauthamer, 1984; Stewart, 1978), and reference to the fixed and cleared image was made in analyzing the structure of the cell. In one experiment, the cell morphology was further analyzed by reconstruct- ing the neuron from optical sections taken every $10 \mu \mathrm{m}$ though the cleared ganglion. A comparison of these images with those made using horseradish peroxidase or cobalt suggests that we may be missing the finest processes. However, this should not affect any of the conclusions of this paper.

\section{Results}

Spike initiation zone in the axon. When a "marginal" cell was depolarized with a current pulse in the soma, it was often observed that the location of the earliest timeto-peak of the action potential (recorded optically) was not in the soma but rather in the axon in the region 100 to $200 \mu \mathrm{m}$ from the cell body. An example is shown in Figure $1 B$ (upper). This result shows that the spike initiated in this zone and propagated orthodromically down the axon and antidromically back to the soma. Figure $1 C$ shows that the time-to-peak for a hyperpolarizing pulse also stimulated in the soma increases with distance from the soma as expected for an electrotonically propagating pulse. This result serves as an internal control for our timing measurements.

Figure $1 B$ (lower) from another neuron shows that the somatic spike is broader than the spike in the initiating zone. In addition, when the cell was stimulated antidromically via the antennular nerve, the action potential recorded electrically in the soma did not overshoot $0 \mathrm{mV}$. These observations are most easily explained by a cell body which is less excitable and has a higher threshold than the axon. However, it is likely that the soma itself is somewhat excitable since the antidromic spike could be made to fail in an all-or-none fashion when the soma was hyperpolarized. This kind of failure has been interpreted as resulting from the impedance mismatch at the soma-axon junction (Tauc, 1962a; Coombs et al., 1957). The actual site of failure has not been experimentally determined and is currently under investigation.

In other experiments, usually when a larger stimulus was given, the soma and initial segment of the axon fired at about the same time (examples are shown in Fig. $1 B$ (lower) and Fig. 2A). In these cases, there was a greater conduction delay between the initial axon segment and region close to the boundary of the ganglion. We interpret these observations as resulting from the spike initiating closer to the soma than in the experiment shown in Figure $1 B$ (upper). The inset in each part of Figure 1 shows a schematic interpretation of the location of the initiation zone in each experiment. In no case was the initiation zone as far away as the boundary of the ganglion.

Spike broadening in the axon. Figure 2 shows selected recordings from four positions on the axon of a cell that was stimulated with a current pulse in the soma. The action potential is significantly shorter in duration in the initiation zone than in the root (Fig. $2 B$ ). After this experiment we moved the visual field of the photodiode away from the ganglion to the nerve and recorded from 10 positions along an axon. Within our error limits there was no variation in the action potential duration along the nerve, and this duration was the same as that measured just inside the boundary of the ganglion (Fig. $2 \mathrm{C}$ ). When the field of view was returned to the original 

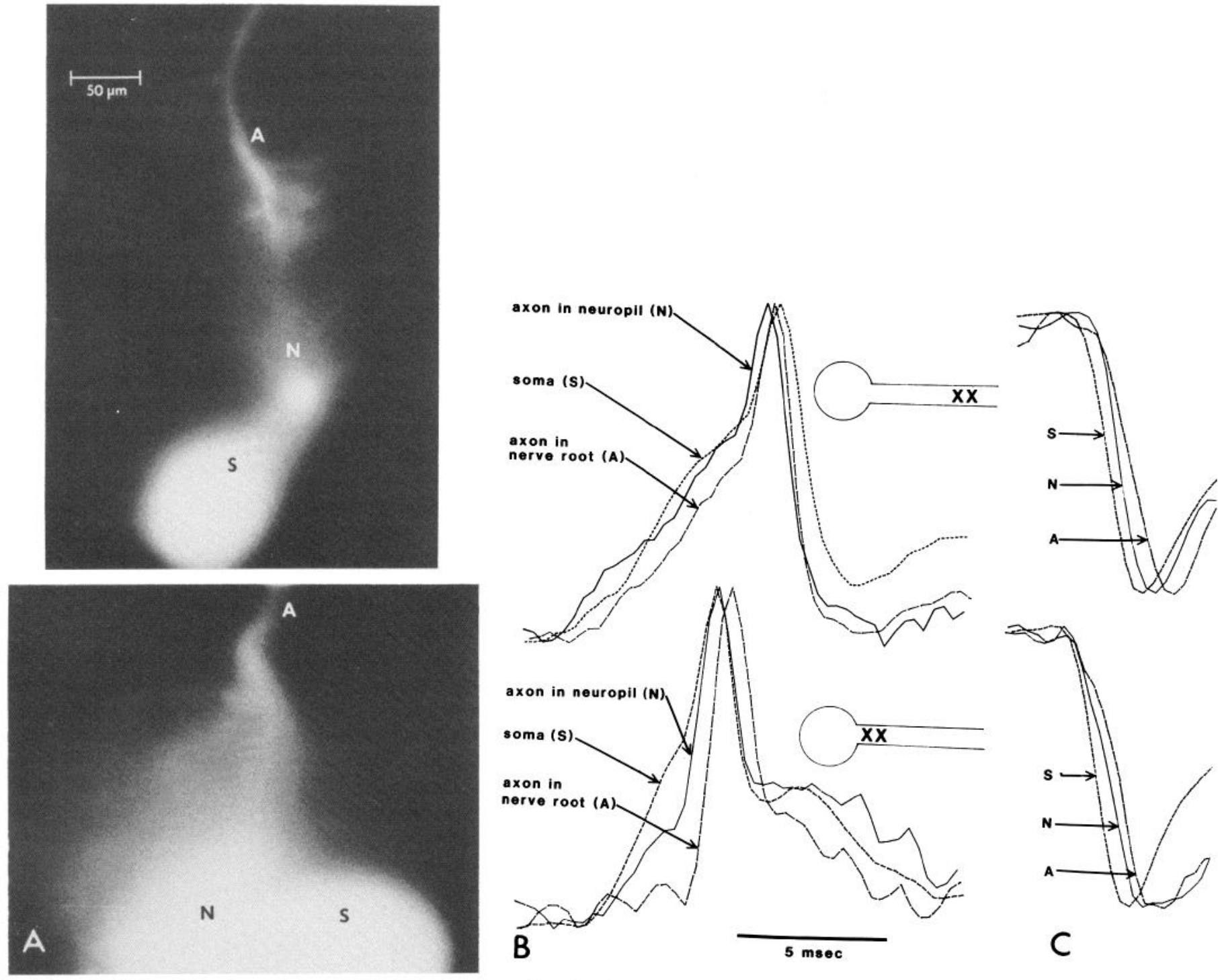

B

$5 \mathrm{msec}$
C

Figure 1. Determination of the spike-initiating zone by the site of the earliest time-to-peak. $A$, Fluorescence photographs of two unfixed cells filled with Lucifer Yellow after optical measurements were made. The fuzziness of the images is due to the fact that the ganglion was not fixed and cleared. However, this allows an accurate match of the optical signals with locations on the cell (Ross and Krauthamer, 1984). Locations $S, N$, and $A$ refer to the soma, neuropil, and axon regions where the recordings in $B$ and $C$ were made. $B$, Simultaneous optical recordings of the action potential in three regions of the cell. All records have been scaled to the same amplitude. Each step on each trace is an interval of 0.333 msec, which corresponds to the sampling rate. Three hundred fifty sweeps were averaged. Data have been digitally filtered at $750 \mathrm{~Hz}$. Records in the upper parts of $B$ and $C$ are from the upper cell in $A$ and records from the lower parts are from the lower cell. Note than in the upper records, the action potential is earliest in the neuropil region, indicated schematically to the right. In the lower records, the action potential is earliest in both the soma and neuropil regions, also shown schematically. $C$, Simultaneous optical recordings of electrotonically propagating hyperpolarizing pulses. The recordings from the site of current injection in the soma have the earliest time-to-peak in both cells.

position, the same difference in durations was observed. This difference was seen many times. Therefore, we conclude that when an action potential is initiated orthodromically in these cells, the spike is shortest in duration in the axon close to the cell body and broadens to a constant duration as it leaves the ganglion.

In these experiments the signal-averaging program for the optical signals used the extracellularly recorded action potential in the antennular nerve as a timing reference. Therefore, the widening of the spike going out of the nerve cannot be attributed to averaging spikes with different conduction velocities or different points of ini- tiation. In addition, the presence of extracellularly recorded action potentials eliminates the possibility that the spike became wider while failing to propagate down the axon.

Dendritic recordings. As shown in the previous paper (Ross and Krauthamer, 1984), we were able to obtain good records of action potentials propagating into the dendrites of the "marginal cells" using signal-averaging. In most cases, the propagation time for action potentials along the dendritic processes (typical length, $160 \mu \mathrm{m}$ ) was less than $0.3 \mathrm{msec}$ and the delay in time-to-peak for a 2-msec-wide hyperpolarizing pulse was less than 0.6 
A

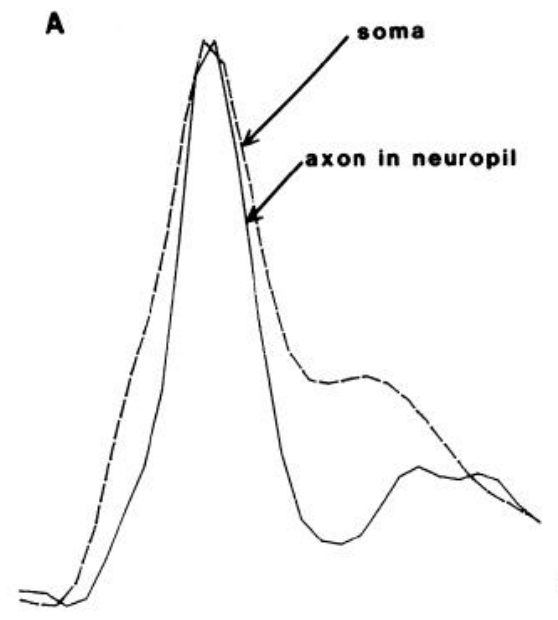

B

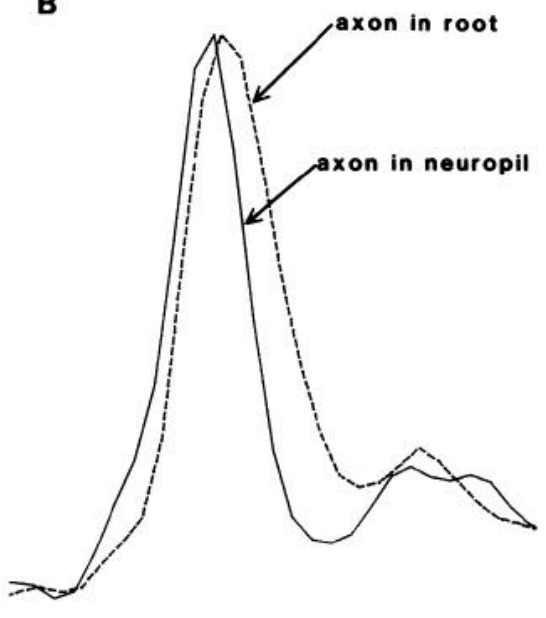

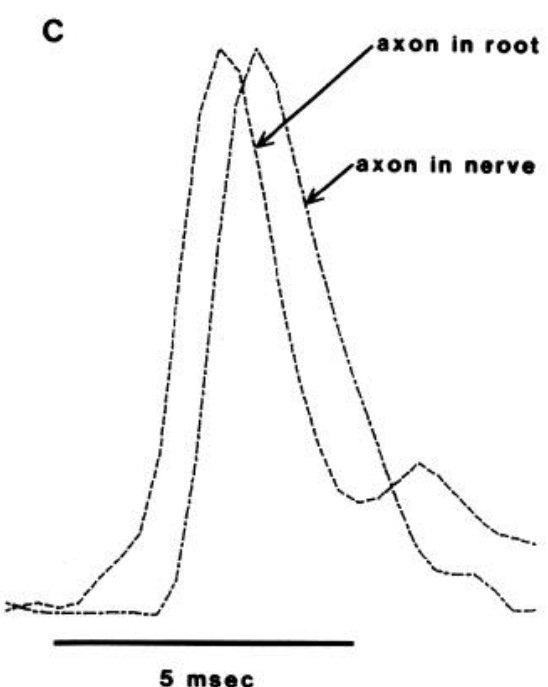

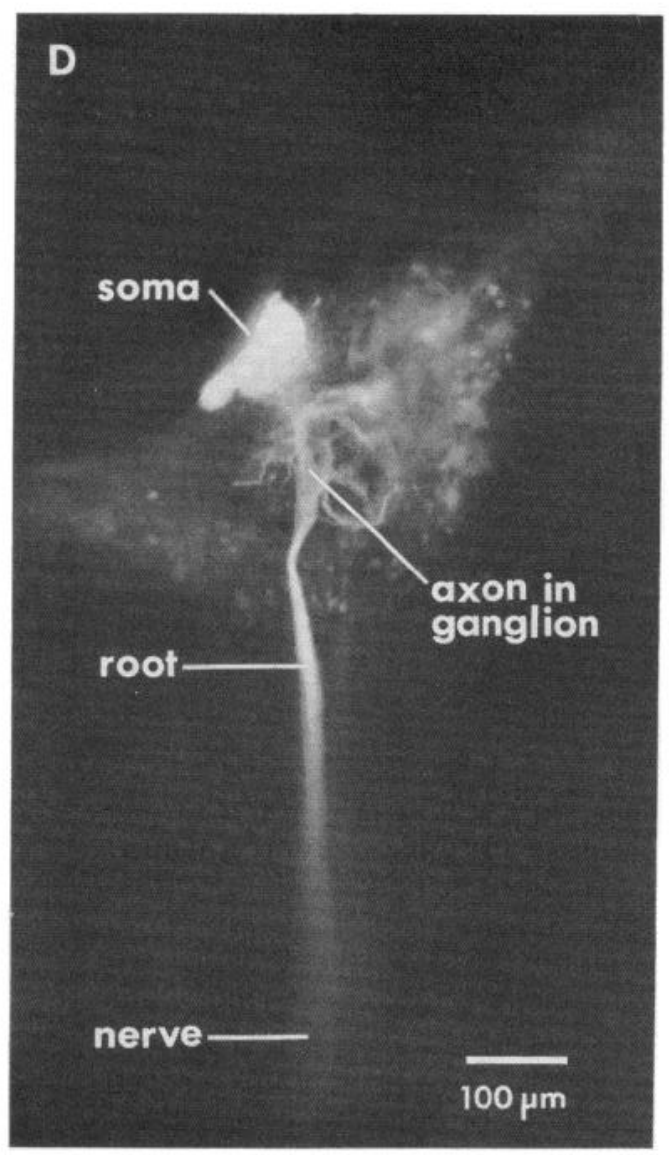

Figure 2. Variation in action potential width in different parts of the cell. The action potential was evoked by current injection into the cell body of a marginal cell (stimulus duration indicated by the time bar at the bottom of the figure), and four records from different positions are shown. The action potential is shortest in duration in the region of the axon within the ganglion where there are many branches. $A$ compares the action potential from the axon within the ganglion to the somatic action potential. The somatic action potential is broader. $B$ shows that the axon within the root has a broader action potential than the axon in the ganglion. This broader action potential is maintained along the nerve $(C)$. The recordings from the soma, axon in ganglion, and root were made simultaneously ( 350 sweeps were averaged). The recording from the nerve ( 250 sweeps averaged) was made immediately after (with the same stimulus parameters), and this was followed by a repeat of the first sequence to be certain that action potential duration did not change with time. $D$ shows the fixed and cleared ganglion after the cell has been filled with Lucifer Yellow. 
msec, suggesting that the electrotonic length of these processes was short. This fact, together with the limited time resolution of our sampling electronics, made it difficult to determine whether the propagation into the dendrites was active or passive (see "Discussion"). However, clear examples of each kind of propagation were obtained.

Active dendrites. Three different kinds of experiments showed regenerative activity in dendrites.

In two experiments on two neurons, we found that when current was injected into the soma, action potentials appeared first in the dendritic region. Figure 4 shows the results of one of these experiments. The earlier timeto-peak in the dendrite $(D)$ shows that the spike must be initiated in this region and hence this region contains active membranes. Because the photodiode detects signals from a field containing more than one process we cannot assign the action potential to a specific dendrite. However, since the action potential was initiated in this region, at least one of the dendrites must be excitable.

Action potentials with a similar early time-to-peak as in the dendritic processes were also recorded from photodiodes in position $A$ in Figure $3 a$, which were located over the axon of the cell within the ganglion. Because this region is neither electrotonically nor physically close to the active dendrites (as demonstrated by the delay of the spread of the hyperpolarizing pulse into the dendrites (Fig. $4 b$ ) and the reconstruction of the cell morphology in Fig. 3c), we conclude that the action potential was simultaneously and separately initiated in the two regions of the cell. The signals in the dendritic region cannot be due to scattering from the axon even though the time courses of the action potentials are similar. This is because the hyperpolarizing pulses, which would be equally affected by scattering, are clearly different in time course in the two regions.

The second experiment is shown in Figure 5. In this case, the soma was first depolarized with a pulse strong enough to make the cell fire and then was stimulated with a subthreshold depolarizing pulse. Figure $5 B$ shows recordings of the subthreshold pulse from four positions along the dendritic process. The amplitudes have been scaled so that the action potentials are all the same height. With this adjustment, and even though the records are noisy, it is clear that the amplitude of the subthreshold pulse declines along the process. The most likely interpretation is that the action potential propagates actively, while the subthreshold pulse propagates passively and decrements. If the action potential propagated passively, then it would have decremented even more rapidly than the subthreshold pulse since the membrane capacitance would have more strongly reduced the faster rising spike. In addition, possible spike jitter or occasional branch point failure of the action potential, which might affect amplitude measurements (see Ross and Krauthamer, 1984), would give results in the opposite direction to those which were observed in this case.

The same preparation was used to give another demonstration of dendritic excitability. When the cell was depolarized sufficiently to evoke double action potentials, the amplitude of the second action potential along the process became smaller than the first. Yet the amplitudes of the two spikes along the axon were equal (Fig. $5 \mathrm{C}$ ). The most likely explanation for this observation is that the two spikes propagate actively into the dendrite, but the second one appears to decline in amplitude because of either occasional branch point failure or a longer refractory period than that found in the axon (see Grossman et al., 1979, for similar records and interpretation). If the action potentials propagated passively into the dendrite, we would expect them both to decline at equal rates and therefore remain with equal amplitudes at each position. In addition, we would expect to see the spikes widen, which was not observed.

Passive dendrites. In addition to these experiments, which indicate that some dendrites are excitable, several records were obtained which demonstrate propagation into dendrites dominated by passive mechanisms. A good example is shown in Figure 6. As in most of the other experiments, the cell was repetitively stimulated with alternating depolarizing and hyperpolarizing pulses, and the resulting absorbance changes were averaged. On the expanded time scale (Fig. 6b) it is clear that the optically recorded spike becomes broader and slower as it propagates into the dendrite. The records were scaled to give equal amplitudes for the hyperpolarizing pulse (Fig. $6 c$ ) (the extreme possibility (see Ross and Krauthamer, 1984) for discussion of amplitude scaling), and we see that the action potential decrements along the dendrite. This result contrasts with that seen in Figure 4. However, the shoulder preceding the action potential, which results from the depolarizing stimulus, does not decrement faster than the hyperpolarizing pulse. The simplest explanation of this experiment is that the action potential did not propagate actively into this process. Another possibility is that the spike propagated actively along the many branches of the dendrite in the field of each photodiode element, but with varying velocities. 'I'he resulting dispersion in propagation time would give the broad, lower amplitude signals observed. We think this possibility is less likely than passive propagation for two reasons. (a) Individual processes with slower velocities would have smaller diameters, and therefore the signals for them would be smaller. As a result, unless there are numerous small processes, the average optical signal is likely to be dominated by the larger processes with faster conduction velocities and little dispersion over the short distance of these processes. (b) In other experiments, where the morphology of the dendritic arborization was similar to that of this experiment, we did not see significant broadening of the action potential or reduction of amplitude.

\section{Discussion}

In the previous paper (Ross and Krauthamer, 1984), we demonstrated that optical techniques could be used to record potential changes simultaneously at many identified positions on a neuron. In addition, we showed that the timing and spatial resolution of these optical signals could be used to analyze the propagation of physiological signals within an individual cell. 

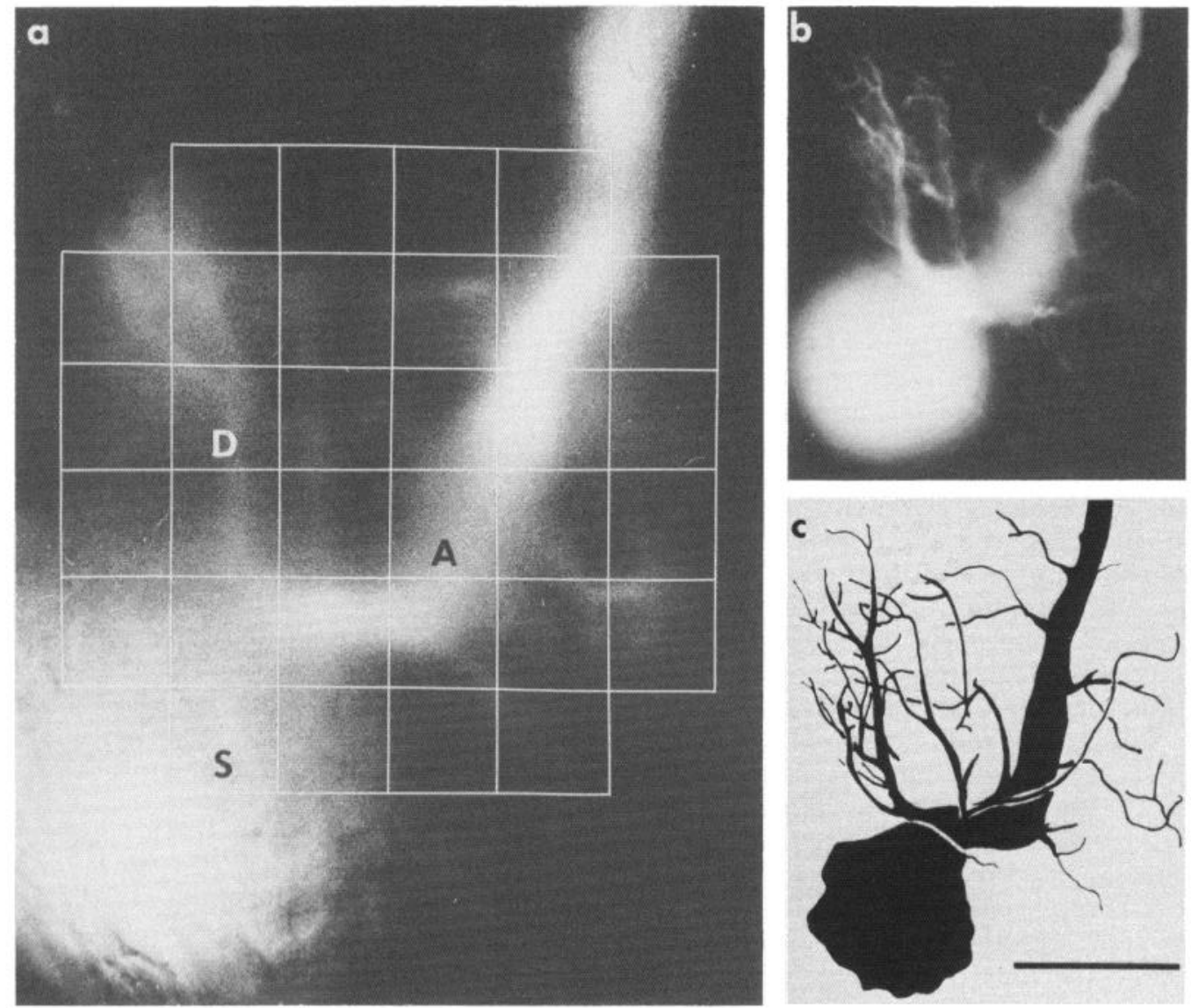

Figure 3. Lucifer Yellow-filled cell used in an experiment to demonstrate excitable dendrites (see Fig. 4 for records obtained from this cell). $a$, Unfixed ganglion viewed with fluorescence optics. The overlay shows the positions of the elements of the photodiode array. $S, A$, and $D$ refer to the locations of the recordings shown in Fig. 4 and indicate soma, axon, and dendrite, respectively. $b$, Fluorescence photograph of the fixed and cleared ganglion. The details of the processes are more visible. $c$, Reconstruction of the same cell based on a series of fluorescence photographs taken every $10 \mu \mathrm{m}$ through the ganglion. Total thickness of the cell in this field of view is about $100 \mu \mathrm{m}$. Scale bar for $b$ and $c$ is $100 \mu \mathrm{m}$.

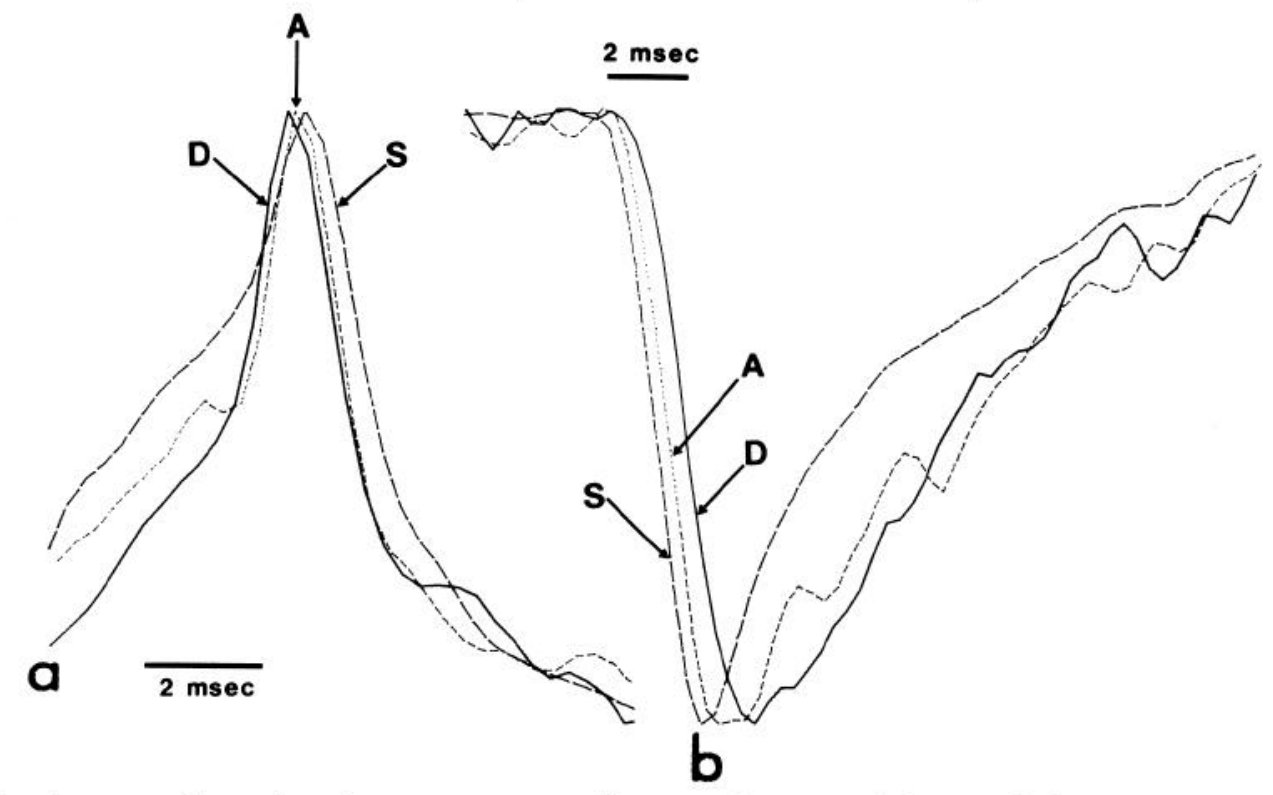

Figure 4. a, Simultaneous absorption changes corresponding to action potentials recorded on photodiodes postitioned over the soma $(S)$, dendritic field $(D)$, and axon $(A)$ (see Fig. 3). The optical signal was sampled every $0.333 \mathrm{msec}$ (the duration of each segment on the traces) and 350 sweeps were averaged. The duration of the depolarizing stimulus was 7 msec. Note that the dendritic signal is earlier and narrower than the one from the cell body. $b$, Absorption changes corresponding to 2 msec (time bar) hyperpolarizing pulses at the same three positions. The pulse in the dendrites is more delayed than that over the axon as expected for electrotonic spread into a narrower process. The difference between the dendritic and axonal traces is evidence that these two photodiodes recorded signals from areas which were electrotonically separated. 

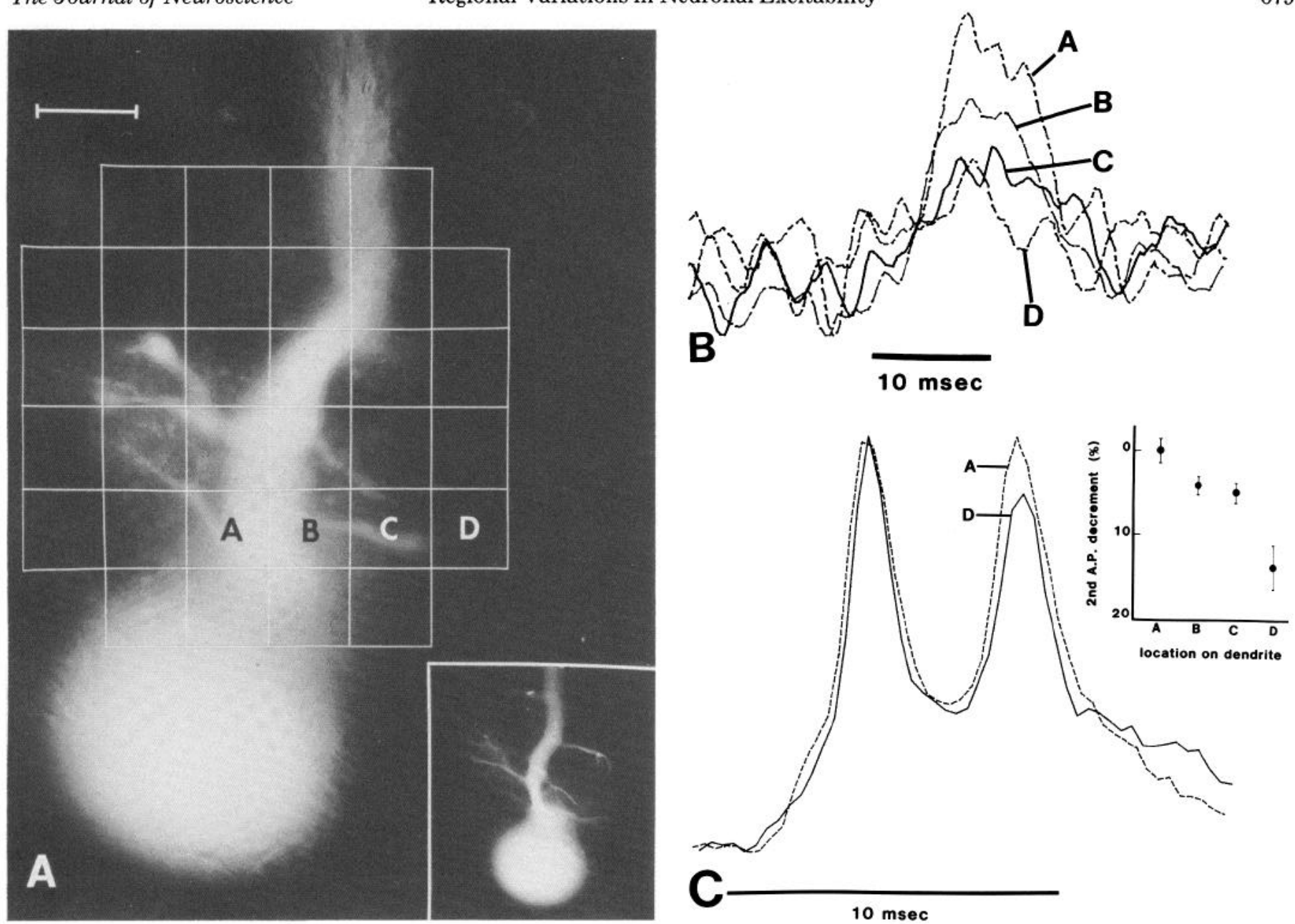

Figure 5. Two other demonstrations of active dendrites. A, Fluorescence photograph of an unfixed ganglion with a cell filled with Lucifer Yellow. The inset shows the cell after fixing and clearing the ganglion. Each box is $40 \mu \mathrm{m} \times 40 \mu \mathrm{m}$, and the positions $A, B, C, D$ refer to the locations of the recordings in $B$ and $C$. The process does not appear to extend into box $D$, but reference to the inset shows that there was a dendrite in this region. $B$, Recordings of optical signals produced by a subthreshold stimulus in the soma given during the time shown on the bar. The amplitudes have been scaled to give equal heights to action potentials along the process produced by stimulating pulses just preceding the subthreshold pulses (not shown). Three hundred sweeps were averaged. Data have been digitally filtered at $350 \mathrm{~Hz}$. The amplitude of the subthreshold pulse declines more rapidly than the amplitude of the action potential. $C$, Recordings of optical signals produced by a somatic stimulus strong enough to make the cell double pulse. The amplitude has been scaled to make the first action potential have the same amplitude at each position along the dendrite. The second pulse at position $D$ has declined in amplitude faster than the first action potential. The inset shows the percentage decline at each position. The error bars indicate 1 SEM recorded during a time period when the cell was not stimulated.

In the results reported here, we have used three kinds of experiments to examine excitability in different regions of these motoneurons: (1) a comparison of the propagation of active and passive potential changes, (2) the propagation of double pulses, and (3) the location of the earliest time-to-peak of an action potential. The timing comparison is the kind of measurement that the optical technique can make most reliably. Unfortunately, we were limited in our measurements to a resolution of $0.333 \mathrm{msec}$ which was determined by the fact that we were sampling 32 channels using an analog-to-digital convertor with a maximum conversion rate of $100 \mathrm{kHz}$. In the previous paper (Ross and Krauthamer, 1984), we showed that better timing resolution could be obtained when looking at the rising phase of a uniformly propagating action potential in an axon. But within the ganglion, where nonuniform excitability and varying dimen- sions can make the action potential have different shapes and amplitudes in different regions, peak timing is more reliable.

The dendrites are only 100 to $200 \mu \mathrm{m}$ long in these "marginal" cells of the supraesophageal ganglion, and the optical signals are dominated by events in the largest diameter branches which have the longest space constants. As a result, the space constant for the events we recorded was probably comparable to or greater than the length of the process. This fact, combined with the above mentioned timing resolution, made it difficult for us to observe propagation delays in many experiments. In addition, the signal-to-noise ratio in the experiments was not always large enough to clearly determine the timeto-peak. Finally, even in those experiments where the timing resolution and signal-to-noise ratio were adequate, the quality of the Lucifer Yellow fill was some- 

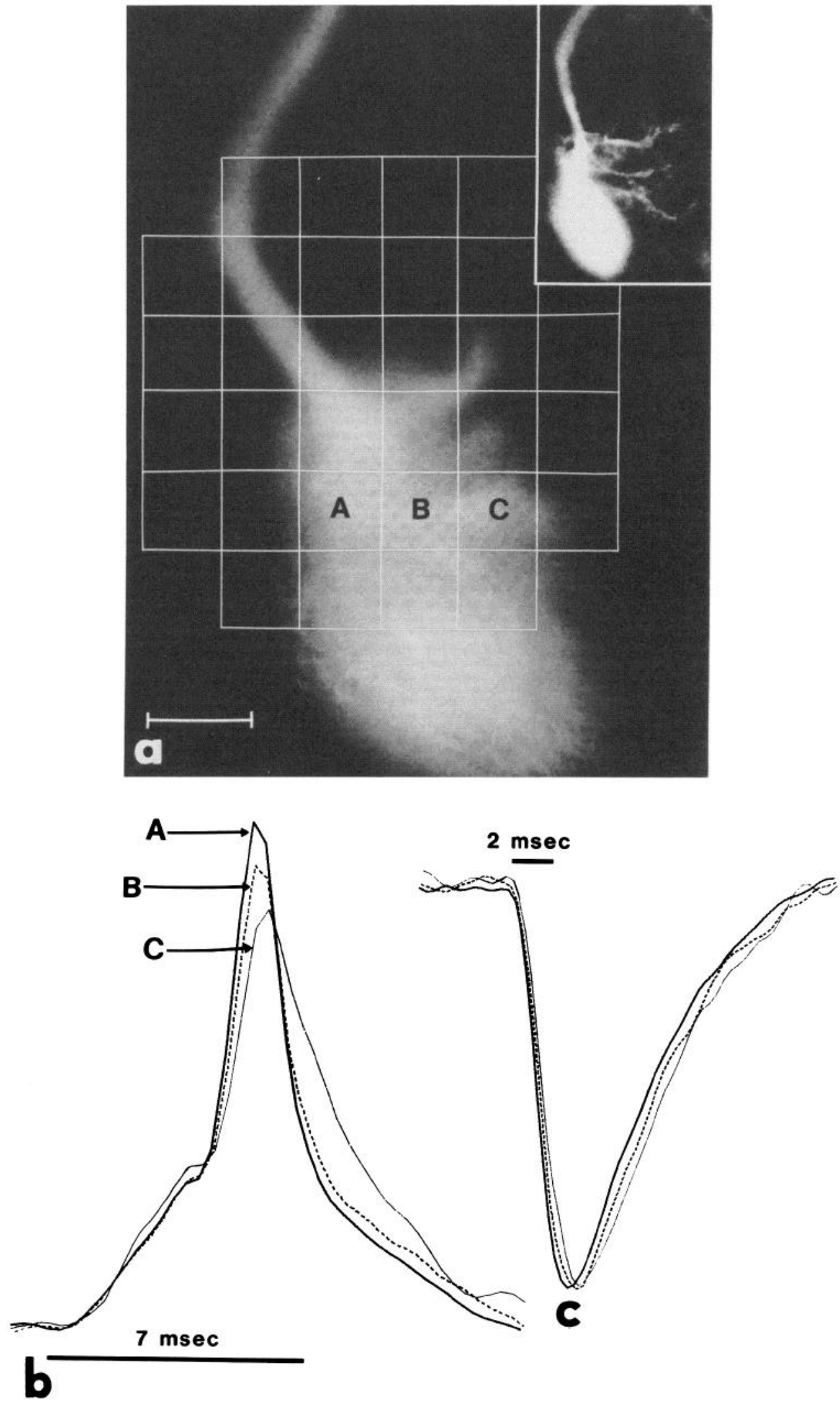

Figure 6. Demonstration of a passive dendrite. $a$, Fluorescence photograph of an unfixed ganglion with a cell filled with Lucifer Yellow. The scale is $60 \mu \mathrm{m}$. The inset shows the fixed and cleared cell. $b$, Optical recordings of a propagating action potential along a dendrite. $A, B$, and $C$ refer to positions along the dendrite shown in $a$. The action potential amplitudes have been scaled so that the simultaneously recorded signals from a hyperpolarizing pulse $(c)$ have the same amplitude. Two hundred fifty trials were averaged. Data have been digitally filtered at $750 \mathrm{~Hz}$. The timings of the current injections are indicated by the time bars. Note that the time scale is smaller in $c$ than in $b$. 
times too poor to make a good assignment of the signals to positions on the cell. Therefore, only a small percentage of experiments satisfied all criteria for an accurate determination of the location of spike initiation.

Spike initiation zone in the axon. When the marginal cell is stimulated with current in the soma, the earliest time-to-peak and hence the initiation point of the action potential is located in the axon within the neuropil. This location is variable from cell to cell and also depends on the stimulus strength. The best explanation for these results is that all of the axon and soma are excitable, but the threshold is lower in the axon. An intense stimulus can directly excite the soma, making it the location of the earliest spike. Lower intensities first excite the axon and the resulting spike propagates in both directions. This picture is consistent with that developed for Aplysia motoneurons (Tauc, 1962a, b) and for locust motoneurons (Gwilliam and Burrows, 1980). But it differs from the view of motoneurons in the cockroach (Pearson and Fourtner, 1975) and in the crab (Sandeman, 1969) where the axonal segment within the ganglion appears to be unexcitable.

The location of the axonal spike initiation zone within the ganglion is also likely to apply to action potentials excited by synaptic activation since almost all of the dendritic processes connect to this part of the cell (Fig. $3 c)$. It is interesting that this region seems to be the location where the action potential is narrowest. This could result from an increased density of voltage-dependent channels in this region. The cable properties of the cell cannot be responsible for this narrowing, since the axon does not taper in this region and processes connecting to the axon would tend to be sinks for the regenerative current and would lead to broader spikes (Goldstein and Rall, 1974), the opposite of what was observed.

This narrowing of the action potential in the neuropil region of the ganglion has not, to our knowledge, been described before. This may be because previous experiments only recorded from one or two sites along the axon, and it is likely that broadening, if observed, may have been attributed to electrode damage.

Dendritic spikes. We have found regenerative activity in dendrites using three different kinds of experiments. The example shown in Figure 5 shows excitability by initiating a spike in a dendritic process. This kind of result, although a clear demonstration, was unusual because in most cases the axon region fired first when the soma was stimulated. The second demonstration, showing differential decrement of action potentials and subthreshold pulses into a process, was also difficult to obtain, often because most processes were too short to cause a significant electrotonic decrement. The third demonstration, using the failure of the second of two action potentials, had the same problem: the failure could only be demonstrated clearly for processes with a long electrotonic length. As a result, we were not able to decide whether most of the processes we examined were excitable even though good optical recordings were obtained from them.

Some experiments indicated that action potentials propagated passively into dendritic processes. In others, it was suggested that the propagation into the dendrites might be blocked at branch points or have a higher level of inactivation. It may be that the dendrites, although excitable, were not as excitable as the axon, especially the initial segment in the neuropil. Another possibility is that the dendrites are heterogeneous, some excitable and some not, or excitable for some fraction of their length (Nelson and Burke, 1967). In principle, the optical recording technique used here can help decide among these possibilities because it has the unique advantage of being able to examine all cell processes simultaneously. But to achieve this goal, we will require improvements in the signal-to-noise ratio and time and spatial resolution beyond those described here. It is also possible that these problems could be examined more completely with the current level of technology using a preparation with longer dendrites and shorter electrotonic lengths.

\section{References}

Calabrese, R. L., and D. Kennedy (1974) Multiple sites of spike initiation in a single dendritic system. Brain Res. 82: 316321.

Coombs, J. S., P. R. Curtis, and J. C. Eccles (1957) The interpretation of spike potentials in motoneurons. J. Physiol (Lond.) 139: 198-231.

Fatt, P. (1957) Electrical potentials occurring around a neurone during its antidromic activation. J. Neurophysiol. 20: 27-60.

Fuortes, M. G. F., K. Frank, and M. C. Becker (1957) Steps in the production of motoneurone spikes. J. Gen. Physiol. 40: $735-752$.

Furshpan, E. J., and T. Furukawa (1962) Intracellular and extracellular responses of the several regions of the Mauthner cell of the goldfish. J. Neurophysiol. 25: 732-771.

Goldstein, S. S., and W. Rall (1974) Changes in action potential shape and velocity for changing core conductor geometry. Biophys. J. 14: 731-757.

Grossman, Y., I. Parnas, and M. E. Spira (1979) Differential conduction block in branches of a bifurcating axon. J. Physiol. (Lond.) 295: 283-305.

Gwilliam, G. F., and M. Burrows (1980) Electrical characteristics of the membrane of an identified insect motor neurone. J. Exp. Biol. 86: 49-61.

Krauthamer, V., and W. N. Ross (1982) Dendrites of a barnacle neuron are excitable: Determination with voltage-sensitive dye. Soc. Neurosci. Abstr. 8: 685 .

Llinas, R. (1975) Electroresponsive properties of dendrites in central neurons. Adv. Neurol. 12: 1-13.

Llinas, R., and C. Nicholson (1971) Electrophysiological properties of dendrites and somata in alligator Purkinje cells. J. Neurophysiol. 34: 532-551.

Llinas, R., and M. Sugimori (1980) Electrophysiological properties of in vitro Purkinje cell dendrites in mammalian cerebellar slices. J. Physiol. (Lond.) 305: 197-213.

Nelson, P. G., and R. E. Burke (1967) Delayed depolarization in cat spinal motoneurons. Exp. Neurol. 17: 16-26.

Nelson, P. G., and K. Frank (1964) Extracellular potential fields of single spinal motoneurons. J. Neurophysiol. 27: 913927.

Pearson, K. G., and C. R. Fourtner (1975) Nonspiking interneurons in walking system of the cockroach. J. Neurophysiol. 38: $33-52$. 
Purpura, D. P. (1967) Comparative physiology of dendrites. In The neurosciences: A Study Program, G. C. Quarton, T. Melnechuk, and F. O. Schmitt eds., pp. 372-393, The Rockefeller University Press, New York.

Ross, W. N., and V. Krauthamer (1984) Optical measurements of potential changes in axons and processes of neurons of a barnacle ganglion. J. Neurosci. 4: 659-672.

Sandeman, D. C. (1969) The site of synaptic activity and impulse initiation in an identified motoneuron in the crab brain. J. Exp. Biol. 50: 771-784.

Spencer, W. A., and E. R. Kandel (1961) Electrophysiology of hippocampal neurons. IV. Fast prepotentials. J. Neurophysiol. 24: 272-285.
Stewart, W. W. (1978) Functional connections between cells are revealed by dye-coupling with a highly fluorescent naphthalimide tracer. Cell 14: 741-759.

Takeda, K., and D. Kennedy (1965) The mechanism of discharge pattern formation in crayfish interneurons. J. Gen. Physiol. 48: 435-453.

Tauc, L. (1962a) Site of origin and propagation of spike in the giant neuron of Aplysia. J. Gen. Physiol. 45: 1077-1097.

Tauc, L. (1962b) Identification of active membrane areas in the giant neuron of Aplysia. J. Gen. Physiol. 45: 1099-1115.

Wong, R. K. S., D. A. Prince, and A. I. Basbaum (1979) Intradendritic recordings from hippocampal neurons. Proc. Natl. Acad. Sci. U. S. A. 76: 986-990. 